\title{
Red Flags for Maltese Adults with Congenital Heart Disease: Poorer Dental Care and Less Sports Participation Compared to Other European Patients-An APPROACH-IS Substudy
}

\author{
Maryanne Caruana ${ }^{1}\left(\mathbb{D} \cdot\right.$ Silke Apers $^{2,3} \cdot$ Adrienne H. Kovacs $^{4} \cdot$ Koen Luyckx $^{5} \cdot$ Corina Thomet $^{6} \cdot$ Werner Budts $^{7}$. \\ Maayke Sluman ${ }^{8} \cdot$ Katrine Eriksen $^{9}$ Mikael Dellborg ${ }^{10,11} \cdot$ Malin Berghammer $^{12,13} \cdot$ Bengt Johansson $^{14}$. \\ Alexandra Souf ${ }^{15}$ - Edward Callus ${ }^{16}$ - Philip Moons ${ }^{2,10} \cdot$ Victor Grech $^{17}$ - On behalf of the APPROACH-IS \\ consortium and the International Society for Adult Congenital Heart Disease (ISACHD)
}

Received: 18 November 2016 / Accepted: 18 March 2017 / Published online: 24 March 2017

(C) Springer Science+Business Media New York 2017

\begin{abstract}
Studies in recent years have explored lifestyle habits and health-risk behaviours in adult congenital heart disease (ACHD) patients when compared to controls. The aim of this study was to investigate differences in lifestyle habits between Maltese and other European ACHD patients. Data on alcohol consumption, cigarette smoking, substance misuse, dental care and physical activity collected in 2013-2015 during "Assessment of Patterns of Patient-Reported Outcomes in Adults with Congenital Heart disease-International Study" (APPROACH-IS) were analysed. Responses from 119 Maltese participants were
\end{abstract}

Collaborators of the APPROACH-IS consortium and the International Society for Adult Congenital Heart Disease (ISACHD) are listed in "Acknowledgements".

Maryanne Caruana

caruana.maryanne@gmail.com

1 Department of Cardiology, Mater Dei Hospital, Birkirkara Bypass, Msida MSD 2090, Malta

2 Department of Public Health and Primary Care, KU Leuven University of Leuven, 3000 Leuven, Belgium

3 Department of Development and Regeneration, KU Leuven University of Leuven, 3000 Leuven, Belgium

4 Knight Cardiovascular Institute, Oregon Health \& Science University, Portland, OR, USA

5 School Psychology and Child and Adolescent Development, KU Leuven - University of Leuven, 3000 Leuven, Belgium

6 Center for Congenital Heart Disease, University Hospital Bern, Bern, Switzerland

7 Division of Congenital and Structural Cardiology, University Hospitals Leuven, KU Leuven - University of Leuven, 3000 Leuven, Belgium

8 Department of Cardiology, Academic Medical Center, Amsterdam, The Netherlands compared to those of 1616 participants from Belgium, France, Italy, Norway, Sweden, Switzerland and the Netherlands. Significantly fewer Maltese patients with simple (Maltese $84.1 \%$ vs. European $97.5 \%, p<0.001$ ) and moderately complex CHD (Maltese $83.6 \%$ vs. European $97.4 \%$, $p<0.001$ ) brushed their teeth daily. Only $67.2 \%$ of Maltese with moderately complex disease had dental reviews in the previous year compared to $80.3 \%$ of Europeans $(p=0.02)$. Maltese patients with simple (Maltese $31.8 \%$ vs. European $56.1 \%, p=0.002$ ) and moderately complex lesions (Maltese $30.0 \%$ vs. European $59.2 \%, p<0.001$ ) performed less regular sport activities. Comparison by country showed Maltese patients to have significantly poorer tooth brushing and sports participation than patients from any other

9 Oslo University Hospital, Rikshospitalet, Oslo, Norway

10 Institute of Medicine, The Sahlgrenska Academy at University of Gothenburg, Gothenburg, Sweden

11 Adult congenital heart unit, Sahlgrenska University Hospital/ Östra and Institute for Medicine, The Sahlgrenska Academy at University of Gothenburg, Gothenburg, Sweden

12 Department of Health Science, University West, Trollhättan, Sweden

13 Centre for Person-Centred Care (GPCC), University of Gothenburg, Gothenburg, Sweden

14 Department of Clinical Medicine and Public Health, Umeå University, Umeå, Sweden

15 Hospital Louis Pradel, Lyon, France

16 Clinical Psychology Unit, IRCCS Policlinico San Donato, San Donato Milanese, Italy

17 Department of Paediatrics, University of Malta Medical School, MSD 2090 Msida, Malta 
participating country. Alcohol consumption, cigarette smoking and substance misuse were not significantly different. This study highlights lifestyle aspects that Maltese ACHD patients need to improve on, which might not be evident upon comparing patients to non-CHD controls. These findings should also caution researchers against considering behaviours among patients in one country as necessarily representative of patients on the larger scale.

Keywords Congenital heart disease $\cdot$ Health behaviour . Lifestyle $\cdot$ Risk factors

\section{Introduction}

The majority of children born with congenital heart disease (CHD) in the current era are expected to survive into adulthood [1]. Adults with congenital heart disease (ACHD), like other adults with special health care needs, may be equally likely or even more prone to engage in healthrisk behaviours compared to their peers [2]. Furthermore, unhealthy habits could have even more deleterious effects in these patients, especially those with more complex CHD lesions [3]. Several European studies have explored lifestyle habits and health-risk behaviours in CHD patients in recent years, mainly by comparing habit uptake between ACHD patients and non-CHD controls, and the results are mixed. Studies by Zomer et al. from the Netherlands [4] and Moons et al. from Belgium [5] reported a healthier lifestyle among ACHD patients when compared to the general population. Sandberg et al. [6] reported physical activity levels among CHD patients in Sweden to be similar to those in the general population. Janssens et al. [7] from Belgium found moderate to good overall health behaviour in a cohort of 429 young CHD people. Overgaard et al. [8] reported similar substance use, cigarette smoking and dental care in a group of Danish patients with single ventricle physiology when compared to matched controls but the patient group reported less physical activity.

Malta is a small country in Southern Europe with a population of around 425,000, and a member of the European Union since 2004. The native language is Maltese, though English is a second official language in several institutions and most people have good English language proficiency. Its health care system is funded through taxation and national insurance, and specialised services are provided in one main teaching hospital. ACHD services were established in the late 1990s. A formal transition process from paediatric care was instituted in late 2015. In 2013-2014, the lifestyle habits of Maltese ACHD patients were investigated by comparing patterns of tobacco smoking, alcohol consumption, substance misuse, dental reviews and physical activity in ACHD patients under clinical follow-up with age-matched and sex-matched subjects from the general Maltese population. Results indicated that although ACHD patients smoked less and attended dental reviews more regularly, they were significantly less physically active than their general population peers irrespective of CHD lesion severity [9].

The aim of the current study was to investigate possible differences in uptake of these lifestyle habits among Maltese ACHD patients when compared to patients from other European centres by utilising data collected during the "Assessment of Patterns of Patient-Reported Outcomes in Adults with Congenital Heart disease-International Study" (APPROACH-IS).

\section{Materials and Methods}

\section{Study Protocol}

APPROACH-IS is a cross-sectional study conducted in partnership with the International Society of Adult Congenital Heart Disease (ISACHD) during which data were collected from 15 countries from 5 continents between April 2013 and March 2015 [10]. Patients were eligible for inclusion if diagnosed with CHD before the age of 10 years, if 18 years of age or older, if under follow-up in a CHD centre or included in a national/regional database and if in possession of the physical, cognitive and language capabilities to answer the self-reporting questionnaires. Patients with previous heart transplantation, primary pulmonary hypertension or cognitive impairment were excluded. Self-reporting questionnaire booklets consisting of different validated and reliability-tested standardised questionnaires could either be sent by surface mail to randomly selected ACHD patients from the centre's database or be given directly to patients at outpatient clinics. Surveys investigated several patient-reported outcomes, including health behaviours which were analysed using the HealthBehaviour Scale-Congenital Heart Disease (HBS-CHD) [11]. Medical records of study participants were reviewed to document the nature of CHD, arrhythmias, number of cardiac procedures, frequency of cardiology follow-up and history of psychiatric disease [10]. CHD severity was classified in accordance with the recommendations of the 32nd Bethesda Conference [12]. Informed consent was obtained from each patient.

In Malta, a completed questionnaire was returned by 119 ACHD patients ('Maltese cohort'). The 'European cohort' consisted of a total of 1616 ACHD responders from 9 participating centres from 7 different European countries (Leuven, Belgium $=276$; Lyon, France $=96$; Milan, Italy $=66$; Oslo, Norway $=174$; Sweden (Gothenburg, Stockholm, Umea) $=471$; Bern, Switzerland $=278$; The Netherlands 
(ConCor registry) $=256$ ). Data from centres in Asia, North and South America and Australia were purposefully excluded from the current study to avoid introducing confounding factors related to major differences in cultural, ethnic and religious backgrounds.

Data from responses to the HBS-CHD section of the APPROACH-IS questionnaire [10] were used to evaluate the 5 following lifestyle habits: (i) alcohol consumption, (ii) cigarette smoking, (iii) substance misuse, (iv) dental care and (v) physical activity. The term "alcohol" referred to any alcoholic beverage and included beer, wine, liquor and coolers. Substance misuse referred to use of one or more of cannabis, 3,4-methylenedioxymethamphetamine ('Ecstasy'), cocaine, hallucinogenic mushrooms, methamphetamine ('Speed'), sleeping pills/sedatives/tranquillizers or other drugs as specified by the responder. Dental care was assessed based on frequency of dental reviews, tooth brushing and dental flossing. Physical activity was assessed in two ways: (a) walking or cycling to work and (b) regular sport participation. During data analysis, responses on each lifestyle habit were collapsed into fewer categories as appropriate to avoid having very small numbers of participants per category.

Preliminary assessment of patient distribution by lesion complexity (i.e. simple, moderate or complex heart lesions) revealed a significantly larger representation of more complex disease among the European cohort (Maltese cohort: simple $37.0 \%$, moderate $51.3 \%$, complex $11.8 \%$ vs. European cohort: simple $31.5 \%$, moderate $47.4 \%$, complex $21.1 \% ; p=0.05)$. In light of the findings from the earlier Malta-based study suggesting mild lesion complexity as a factor associated with higher uptake of tobacco smoking and substance misuse [9], comparison analyses of habit uptake between the two cohorts were performed separately for each level of disease complexity.

\section{Statistical Methods}

In a first analysis, the five lifestyle habits for Maltese participants were compared with those for all participants in the European cohort. For those habits in which a significant difference between the two cohorts was evident, a further analysis was performed by comparing Maltese patients to those from each individual European country. Categorical variables were analysed using Chi-square tests, while in the case of smaller sample sizes, Fisher's Exact test was applied. Shapiro-Wilk testing applied to participant ages showed a non-normal distribution and thus the Mann-Whitney $U$ test was applied. All analyses were performed using SPSS 21 (IBM $^{\circledR}$ SPSS $^{\circledR} 21$, SPSS Inc., Chicago IL, USA). Statistical significance was defined as $p \leq 0.05$ and all tests were two-sided.

\section{Results}

\section{Sample Characteristics}

The basic characteristics of the study participants are summarised in Table 1. There were no significant differences in gender between the two cohorts, although there were more females in the Maltese simple and moderate lesion complexity subgroups. Maltese patients in all three complexity subgroups were significantly younger compared to the respective participants from the European cohort, with the difference in age being most marked in patients with simple and moderately complex lesions. There were significantly more Maltese participants with simple lesions that claimed to have religious leaning (100\%) compared to patients from the European cohort $(89.8 \%)(p=0.05)$.

European participants with lesions of moderate complexity had undergone more cardiac surgeries than patients in the Maltese cohort. Arrhythmias were more common among patients in the European cohort with simple and moderate lesions. Furthermore, a significantly higher proportion of patients with moderately complex CHD in the European cohort had undergone implantation of a cardioverter-defibrillator (ICD) or permanent pacemaker (PPM). On the other hand, medical co-morbidities were significantly more common among Maltese participants with CHD of simple and moderate complexity. Significantly more patients with simple disease in the European cohort $(41.6 \%)$ were being reviewed at least every 2 years compared to those in the Maltese cohort $(11.4 \%)(p<0.001)$, while follow-up for patients with CHD of moderate and great complexity was similar for participants from the two cohorts. Other medical characteristics, including frequency of documented heart failure and hospital admissions, were comparable between the two cohorts across all lesion complexities (Table 1).

\section{Lifestyle Comparison Between Maltese and European Cohorts}

Comparison of lifestyle habits between the two cohorts subdivided by lesion complexity are summarised in Table 2 . Significantly fewer Maltese patients with simple and moderate lesion complexity brushed their teeth at least daily (simple: Maltese $84.1 \%$ vs. European 97.5\%, $p<0.001$; moderate: Maltese $83.6 \%$ vs. European $97.4 \%, p<0.001$ ). Similarly, these Maltese patient subgroups had more erratic dental reviews, with only $67.2 \%$ of Maltese with moderately complex disease having had a dental review in the previous year compared to $80.3 \%$ of European counterparts $(p=0.02)$. Furthermore, $50.0 \%$ of Maltese patients with simple disease had not had dental review in the preceding year, having had one more than 3 years prior to the time of 
Table 1 Comparison of characteristics of participants in the Maltese and European cohorts subdivided by disease complexity

\begin{tabular}{|c|c|c|c|c|c|c|c|c|c|}
\hline \multirow[t]{2}{*}{ Characteristic } & \multicolumn{2}{|c|}{ Simple lesion complexity } & \multirow[t]{2}{*}{$\mathrm{p}$} & \multicolumn{2}{|c|}{ Moderate lesion complexity } & \multirow[t]{2}{*}{$\mathrm{p}$} & \multicolumn{2}{|c|}{ Severe lesion complexity } & \multirow[t]{2}{*}{$\mathrm{p}$} \\
\hline & $\begin{array}{l}\text { Maltese } \\
(n=44)\end{array}$ & $\begin{array}{l}\text { European } \\
(n=509)\end{array}$ & & $\begin{array}{l}\text { Maltese } \\
(n=61)\end{array}$ & $\begin{array}{l}\text { European } \\
(n=766)\end{array}$ & & $\begin{array}{l}\text { Maltese } \\
(n=14)\end{array}$ & $\begin{array}{l}\text { European } \\
(n=341)\end{array}$ & \\
\hline Sex & & & 0.23 & & & 0.52 & & & 0.69 \\
\hline Male & $16 / 44(36.4 \%)$ & $\begin{array}{l}231 / 509 \\
(45.4 \%)\end{array}$ & & $29 / 61(47.5 \%)$ & $\begin{array}{l}397 / 766 \\
(51.8 \%)\end{array}$ & & $7 / 14(50.0 \%)$ & $\begin{array}{l}189 / 341 \\
(55.4 \%)\end{array}$ & \\
\hline Female & $28 / 44(63.6 \%)$ & $\begin{array}{l}278 / 509 \\
(54.6 \%)\end{array}$ & & $32 / 61(52.5 \%)$ & $\begin{array}{l}369 / 766 \\
(48.2 \%)\end{array}$ & & $7 / 14(50.0 \%)$ & $\begin{array}{l}152 / 341 \\
(44.6 \%)\end{array}$ & \\
\hline $\begin{array}{l}\text { Mean age } \\
\quad(\text { years } \pm 1 S D)\end{array}$ & $29.66 \pm 12.01$ & $39.12 \pm 14.48$ & $<\mathbf{0 . 0 0 1}$ & $33.52 \pm 13.36$ & $38.81 \pm 13.67$ & 0.001 & $29.59 \pm 5.23$ & $32.17 \pm 10.56$ & 0.05 \\
\hline $\begin{array}{l}\text { White/Cauca- } \\
\operatorname{sian}^{\mathrm{a}}\end{array}$ & $38 / 39(97.4 \%)$ & $\begin{array}{l}464 / 491 \\
(94.5 \%)\end{array}$ & 0.71 & $55 / 56(98.2 \%)$ & $\begin{array}{l}700 / 744 \\
(94.1 \%)\end{array}$ & 0.36 & $13 / 13(100 \%)$ & $\begin{array}{l}324 / 333 \\
(97.3 \%)\end{array}$ & 0.55 \\
\hline \multicolumn{10}{|c|}{ Religion/spirituality } \\
\hline $\begin{array}{l}\text { Belong to } \\
\text { religion }\end{array}$ & $37 / 37(100.0 \%)$ & $\begin{array}{l}167 / 186 \\
(89.8 \%)\end{array}$ & 0.05 & $45 / 48(93.8 \%)$ & $\begin{array}{l}245 / 276 \\
(88.8 \%)\end{array}$ & 0.44 & $12 / 12(100 \%)$ & $\begin{array}{l}100 / 117 \\
(85.5 \%)\end{array}$ & 0.37 \\
\hline Christianity $^{\mathrm{b}}$ & $37 / 37(100 \%)$ & $\begin{array}{r}145 / 167 \\
(86.8 \%)\end{array}$ & 0.02 & $44 / 45(97.8 \%)$ & $\begin{array}{l}227 / 245 \\
(92.7 \%)\end{array}$ & 0.33 & $12 / 12(100 \%)$ & $87 / 102(85.3 \%)$ & 0.36 \\
\hline \multicolumn{10}{|c|}{ Clinical characteristics } \\
\hline $\begin{array}{r}>3 \text { cardiac } \\
\text { surgeries }\end{array}$ & $0 / 44(0.0 \%)$ & $7 / 359(1.9 \%)$ & 0.35 & $0 / 61(0.0 \%)$ & $60 / 716(8.4 \%)$ & 0.01 & $2 / 14(14.3 \%)$ & $52 / 333(15.6 \%)$ & 0.89 \\
\hline $\begin{array}{r}>3 \text { interven- } \\
\text { tional caths }\end{array}$ & $0 / 44(0.0 \%)$ & $1 / 337(0.3 \%)$ & 0.72 & $0 / 61(0.0 \%)$ & $34 / 562(6.0 \%)$ & 0.07 & $0 / 14(0.0 \%)$ & $39 / 284(13.7 \%)$ & 0.23 \\
\hline $\begin{array}{l}\text { Documented } \\
\text { heart failure }\end{array}$ & $0 / 43(0.0 \%)$ & $20 / 501(4.0 \%)$ & 0.39 & $3 / 61(4.9 \%)$ & $64 / 755(8.5 \%)$ & 0.33 & $0 / 14(0.0 \%)$ & $59 / 339(17.4 \%)$ & 0.14 \\
\hline Arrhythmias & $1 / 44(2.3 \%)$ & $97 / 502(19.3 \%)$ & 0.01 & $6 / 60(10.0 \%)$ & $\begin{array}{l}175 / 762 \\
(23.0 \%)\end{array}$ & 0.02 & $3 / 14(21.4 \%)$ & $\begin{array}{l}155 / 339 \\
(45.7 \%)\end{array}$ & 0.07 \\
\hline $\begin{array}{l}\text { ICD/PPM } \\
\text { implanted }\end{array}$ & $0 / 44(0.0 \%)$ & $25 / 332(7.5 \%)$ & 0.06 & $1 / 61(1.6 \%)$ & $60 / 561(10.7 \%)$ & 0.02 & $2 / 14(14.3 \%)$ & $62 / 266(23.3 \%)$ & 0.74 \\
\hline $\begin{array}{l}\text { Other medical } \\
\text { conditions }\end{array}$ & $23 / 44(52.3 \%)$ & $\begin{array}{l}183 / 497 \\
(36.8 \%)\end{array}$ & 0.04 & $32 / 61(52.5 \%)$ & $\begin{array}{l}281 / 760 \\
(37.0 \%)\end{array}$ & 0.02 & $4 / 14(28.6 \%)$ & $\begin{array}{l}126 / 337 \\
(37.4 \%)\end{array}$ & 0.50 \\
\hline $\begin{array}{l}\text { Admissions in } \\
\text { past year }\end{array}$ & $3 / 44(6.8 \%)$ & $41 / 482(8.5 \%)$ & 0.70 & $4 / 61(6.6 \%)$ & $61 / 751(8.1 \%)$ & 0.81 & $0 / 14(0 \%)$ & $57 / 334(17.1 \%)$ & 0.14 \\
\hline $\begin{array}{l}\text { Clinic visit at } \\
\text { least every } 2 \\
\text { years }\end{array}$ & $5 / 44(11.4 \%)$ & $\begin{array}{l}204 / 490 \\
(41.6 \%)\end{array}$ & $<0.001$ & $41 / 61(67.2 \%)$ & $\begin{array}{l}494 / 745 \\
\quad(66.3 \%)\end{array}$ & 0.90 & $14 / 14(100 \%)$ & $\begin{array}{l}292 / 334 \\
(87.4 \%)\end{array}$ & 0.39 \\
\hline
\end{tabular}

Frequencies for each characteristic are expressed as fractions out of total responses followed by a percentage. Statistically significant differences are shown in bold

${ }^{a}$ Non-white/Caucasian ethnic backgrounds = Middle Eastern/Arabic, Asian, black/African-American, Hispanic/Latino and other ethnicities

${ }^{\mathrm{b}}$ Religions other than Christianity $=$ Buddhism, Hinduism, Islam, Judaism and others

the study compared to only $20.5 \%$ in the European cohort $(p=0.02)$. Maltese patients with simple and moderately complex lesions performed significantly less regular sports activities compared to European patients (simple: Maltese $31.8 \%$ vs. European $56.1 \%, p=0.002$; moderate: Maltese $30.0 \%$ vs. European $59.2 \%, p<0.001)$.

Significantly more European patients with simple CHD (84.8\%) consumed alcohol to any degree compared to their Maltese counterparts $(72.7 \%)(p=0.04)$, though patterns and frequency of alcohol consumption between the two subgroups were not statistically significant. There was a higher proportion of patients from the
European cohort that admitted to substance misuse for all three lesion complexities in the European cohort when compared to the Maltese cohort, but none of the differences reached statistical significance. Cigarette smoking was commoner among Maltese patients with simple CHD (27.3\%) when compared to Europeans with similar lesion complexity (16.2\%), while the opposite was the case for patients with severe CHD lesions with $12.6 \%$ of Europeans admitting to cigarette smoking compared to $7.1 \%$ of Maltese patients. However, none of these differences were statistically significant. 
Table 2 Comparison of lifestyle habits among participants in the Maltese and European cohorts subdivided by disease complexity

\begin{tabular}{|c|c|c|c|c|c|c|c|c|c|}
\hline \multirow[t]{2}{*}{ Lifestyle habit } & \multicolumn{2}{|c|}{ Simple lesion complexity } & \multirow[t]{2}{*}{$\mathrm{p}$} & \multicolumn{2}{|c|}{ Moderate lesion complexity } & \multirow[t]{2}{*}{$\mathrm{p}$} & \multicolumn{2}{|c|}{ Severe lesion complexity } & \multirow[t]{2}{*}{$\mathrm{p}$} \\
\hline & $\begin{array}{l}\text { Maltese } \\
(n=44)\end{array}$ & $\begin{array}{l}\text { European } \\
(n=509)\end{array}$ & & $\begin{array}{l}\text { Maltese } \\
(n=61)\end{array}$ & $\begin{array}{l}\text { European } \\
(n=766)\end{array}$ & & $\begin{array}{l}\text { Maltese } \\
(n=14)\end{array}$ & $\begin{array}{l}\text { European } \\
(n=341)\end{array}$ & \\
\hline \multicolumn{10}{|l|}{ Tobacco smoking } \\
\hline Smokers & $12 / 44(27.3 \%)$ & $82 / 505(16.2 \%)$ & 0.06 & $9 / 61(14.8 \%)$ & $\begin{array}{c}109 / 757 \\
(14.4 \%)\end{array}$ & 0.94 & $1 / 14(7.1 \%)$ & $42 / 334(12.6 \%)$ & 0.55 \\
\hline Daily smokers & $6 / 12(50.0 \%)$ & $44 / 82(53.7 \%)$ & 0.81 & $5 / 9(55.6 \%)$ & $48 / 109(44.0 \%)$ & 0.73 & $0 / 1(0.0 \%)$ & $15 / 41(36.6 \%)$ & 0.45 \\
\hline $\begin{array}{l}\text { Smokers > } 20 \\
\text { cigs dly }\end{array}$ & $2 / 12(16.7 \%)$ & $2 / 82(2.4 \%)$ & 0.08 & $1 / 9(11.1 \%)$ & $5 / 109(4.6 \%)$ & 0.39 & $0 / 1(0.0 \%)$ & $1 / 40(2.5 \%)$ & 0.87 \\
\hline \multicolumn{10}{|l|}{ Alcohol intake } \\
\hline $\begin{array}{l}\text { Consume any } \\
\text { alcohol }\end{array}$ & $32 / 44(72.7 \%)$ & $\begin{array}{l}430 / 507 \\
(84.8 \%)\end{array}$ & 0.04 & $46 / 61(75.4 \%)$ & $\begin{array}{c}646 / 763 \\
(84.7 \%)\end{array}$ & 0.06 & $11 / 14(78.6 \%)$ & $\begin{array}{l}277 / 338 \\
(82.0 \%)\end{array}$ & 0.73 \\
\hline $\begin{array}{l}\text { More than } \\
\text { once/week }\end{array}$ & $9 / 32(28.1 \%)$ & $\begin{array}{c}122 / 430 \\
(28.4 \%)\end{array}$ & 0.98 & $11 / 46(23.9 \%)$ & $\begin{array}{l}200 / 646 \\
(31.0 \%)\end{array}$ & 0.32 & $0 / 11(0.0 \%)$ & $70 / 276(25.4 \%)$ & 0.07 \\
\hline $\begin{array}{l}>4 \text { glasses on } \\
\text { average }\end{array}$ & $6 / 32(18.8 \%)$ & $53 / 430(12.3 \%)$ & 0.28 & $10 / 46(21.7 \%)$ & $\begin{array}{c}101 / 644 \\
(15.7 \%)\end{array}$ & 0.28 & $0 / 11(0.0 \%)$ & $49 / 274(17.9 \%)$ & 0.22 \\
\hline $\begin{array}{l}\geq 6 \text { glasses at } \\
\text { least weekly }\end{array}$ & $0 / 32(0.0 \%)$ & $14 / 430(3.3 \%)$ & 0.61 & $1 / 46(2.2 \%)$ & $28 / 645(4.3 \%)$ & 0.71 & $0 / 11(0.0 \%)$ & $8 / 276(2.9 \%)$ & 0.57 \\
\hline \multicolumn{10}{|l|}{ Substance misuse } \\
\hline $\begin{array}{l}\geq \text { once in past } \\
\text { year }\end{array}$ & $2 / 41(4.9 \%)$ & $57 / 491(11.6 \%)$ & 0.30 & $4 / 57(7.0 \%)$ & $\begin{array}{c}110 / 750 \\
(14.7 \%)\end{array}$ & 0.11 & $0 / 12(0.0 \%)$ & $51 / 327(15.6 \%)$ & 0.23 \\
\hline \multicolumn{10}{|l|}{ Dental care } \\
\hline $\begin{array}{l}\text { Dental review } \\
\text { in past year }\end{array}$ & $29 / 44(65.9 \%)$ & $\begin{array}{l}384 / 505 \\
\quad(76.0 \%)\end{array}$ & 0.14 & $41 / 61(67.2 \%)$ & $\begin{array}{l}613 / 763 \\
(80.3 \%)\end{array}$ & 0.02 & $9 / 14(64.3 \%)$ & $\begin{array}{l}256 / 339 \\
(75.5 \%)\end{array}$ & 0.35 \\
\hline $\begin{array}{l}\text { Last dental } \\
\text { review } \geq 3 \\
\text { years }\end{array}$ & $7 / 14(50.0 \%)$ & $24 / 117(20.5 \%)$ & 0.02 & $7 / 20(35.0 \%)$ & $36 / 145(24.8 \%)$ & 0.33 & $2 / 5(40.0 \%)$ & $17 / 85(20.0 \%)$ & 0.28 \\
\hline $\begin{array}{l}\text { At least daily } \\
\text { tooth brush- } \\
\text { ing }\end{array}$ & $37 / 44(84.1 \%)$ & $\begin{array}{r}472 / 484 \\
(97.5 \%)\end{array}$ & $<0.001$ & $51 / 61(83.6 \%)$ & $\begin{array}{l}719 / 738 \\
(97.4 \%)\end{array}$ & $<0.001$ & $13 / 14(92.9 \%)$ & $\begin{array}{l}320 / 333 \\
(96.1 \%)\end{array}$ & 0.44 \\
\hline $\begin{array}{l}\text { At least daily } \\
\text { dental floss- } \\
\text { ing }\end{array}$ & $3 / 43(7.0 \%)$ & $\begin{array}{l}107 / 486 \\
(22.0 \%)\end{array}$ & 0.02 & $2 / 60(3.3 \%)$ & $\begin{array}{l}178 / 742 \\
(24.0 \%)\end{array}$ & $<0.001$ & $0 / 13(0.0 \%)$ & $57 / 335(17.0 \%)$ & 0.14 \\
\hline \multicolumn{10}{|l|}{ Physical exercise } \\
\hline $\begin{array}{l}\text { Walking/ } \\
\text { cycling to } \\
\text { work }\end{array}$ & $20 / 44(45.5 \%)$ & $\begin{array}{l}220 / 498 \\
(44.2 \%)\end{array}$ & 0.87 & $20 / 59(33.9 \%)$ & $\begin{array}{l}293 / 753 \\
(38.9 \%)\end{array}$ & 0.45 & $5 / 14(35.7 \%)$ & $\begin{array}{l}126 / 334 \\
(37.7 \%)\end{array}$ & 0.88 \\
\hline $\begin{array}{l}\text { Regular } \\
\text { sports activ- } \\
\text { ity }\end{array}$ & $14 / 44(31.8 \%)$ & $\begin{array}{l}282 / 503 \\
(56.1 \%)\end{array}$ & 0.002 & $18 / 60(30.0 \%)$ & $\begin{array}{l}447 / 755 \\
\quad(59.2 \%)\end{array}$ & $<\mathbf{0 . 0 0 1}$ & $4 / 14(28.6 \%)$ & $\begin{array}{c}175 / 333 \\
(52.6 \%)\end{array}$ & 0.08 \\
\hline
\end{tabular}

Frequencies for each characteristic are expressed as fractions out of total responses followed by a percentage. Statistically significant differences are shown in bold

\section{Lifestyle per Country}

A second analysis compared dental health and participation in regular sports activities between all Maltese participants with participants from each European country represented in this study (Table 3). Only $66.4 \%$ of Maltese subjects had undergone a dental review in the preceding year, which was significantly lower than that for subjects from Belgium, the Netherlands and Switzerland. Furthermore, $41.0 \%$ of Maltese subjects that had not visited a dentist in the previous year, admitted not to have had a dental review for at least 3 years, which was significantly more when compared to subjects from Italy, Norway, Switzerland and Sweden. The Maltese cohort was the one with the lowest percentage of subjects that brushed their teeth at least once a day $(84.9 \%)$ and this was significantly less when compared to subjects from each of the other European countries. Similarly, only a very small number of Maltese subjects $(4.3 \%)$ flossed their teeth at least daily, which is significantly less than was seen in subjects from all other European countries apart from France. Only 30.5\% of all Maltese subjects 
Table 3 Comparison of dental care and regular sports participation between Maltese subjects and subjects from each of the other participating European countries

\begin{tabular}{|c|c|c|c|c|c|c|c|c|}
\hline & Malta & Belgium & Netherlands & Italy & Norway & Switzerland & France & Sweden \\
\hline \multicolumn{9}{|l|}{ Dental care } \\
\hline $\begin{array}{l}\text { Dental review } \\
\text { in past } \\
1 \text { year }\end{array}$ & $66.4 \%$ & $\begin{array}{l}86.2 \% \\
\quad(p<0.001)\end{array}$ & $\begin{array}{l}87.0 \% \\
\quad(p<0.001)\end{array}$ & $\begin{array}{l}51.7 \% \\
\quad(p=0.06)\end{array}$ & $\begin{array}{l}72.3 \% \\
\quad(p=0.28)\end{array}$ & $\begin{array}{l}83.5 \% \\
\quad(p<0.001)\end{array}$ & $\begin{array}{l}66.3 \% \\
\quad(p=1.00)\end{array}$ & $72.8 \%(p=0.16)$ \\
\hline $\begin{array}{l}\text { Dental } \\
\text { review }>3 \\
\text { years }^{+}\end{array}$ & $41.0 \%$ & $\begin{array}{l}22.2 \% \\
\quad(p=0.08)\end{array}$ & $\begin{array}{l}37.9 \% \\
\quad(p=0.80)\end{array}$ & $\begin{array}{l}17.9 \% \\
\quad(p=0.04)\end{array}$ & $\begin{array}{l}18.4 \% \\
\quad(p=0.02)\end{array}$ & $\begin{array}{l}17.4 \% \\
(p=0.02)\end{array}$ & $\begin{array}{l}36.7 \% \\
\quad(p=0.71)\end{array}$ & $19.4 \%(p=0.01)$ \\
\hline $\begin{array}{l}\text { At least daily } \\
\text { tooth brush- } \\
\text { ing }\end{array}$ & $84.9 \%$ & $\begin{array}{l}92.9 \% \\
\quad(p=0.01)\end{array}$ & $\begin{array}{l}97.5 \% \\
\quad(p<0.001)\end{array}$ & $\begin{array}{l}98.4 \% \\
\quad(p=0.004)\end{array}$ & $\begin{array}{l}98.3 \% \\
\quad(p<0.001)\end{array}$ & $\begin{array}{l}98.9 \% \\
\quad(p<0.001)\end{array}$ & $\begin{array}{l}96.8 \% \\
\quad(p=0.004)\end{array}$ & $\begin{array}{l}97.8 \% \\
\quad(p<0.001)\end{array}$ \\
\hline $\begin{array}{l}\text { At least daily } \\
\text { dental floss- } \\
\text { ing }\end{array}$ & $4.3 \%$ & $\begin{array}{l}12.2 \% \\
\quad(p=0.02)\end{array}$ & $\begin{array}{l}20.7 \% \\
\quad(p<0.001)\end{array}$ & $\begin{array}{l}20.0 \% \\
\quad(p=0.001)\end{array}$ & $\begin{array}{l}23.0 \% \\
\quad(p<0.001)\end{array}$ & $\begin{array}{l}20.0 \% \\
\quad(p<0.001)\end{array}$ & $3.2 \%(p=0.74)$ & $\begin{array}{l}32.5 \% \\
\quad(p<0.001)\end{array}$ \\
\hline $\begin{array}{l}\text { Regular sports } \\
\text { participation }\end{array}$ & $30.5 \%$ & $\begin{array}{l}45.8 \% \\
\quad(p=0.01)\end{array}$ & $\begin{array}{l}56.2 \% \\
\quad(p<0.001)\end{array}$ & $\begin{array}{l}46.8 \% \\
\quad(p=0.03)\end{array}$ & $\begin{array}{l}65.7 \% \\
\quad(p<0.001)\end{array}$ & $\begin{array}{l}64.7 \% \\
\quad(p<0.001)\end{array}$ & $\begin{array}{l}47.4 \% \\
\quad(p=0.01)\end{array}$ & $\begin{array}{l}59.1 \% \\
\quad(p<0.001)\end{array}$ \\
\hline
\end{tabular}

Responses for each country are expressed as percentages. Numbers in parentheses represent $p$ values with statistically significant differences being shown in bold

${ }^{+}$For patients who had not had a dental review in previous 1 year

claimed regular sports participation and this is significantly less than that reported by subjects from all other European countries.

\section{Discussion}

As more patients born with CHD survive into adulthood, the risk of ACHD patients developing acquired heart disease is increasing. A number of acquired cardiac-related conditions, e.g. hypertension, atherosclerotic disease, diabetes and infective endocarditis (IE) are, at least in part, amenable to lifestyle changes. As a result, there has been increasing interest in health-risk behaviour in the ACHD population worldwide [13]. This is the first study to compare lifestyle habits of Maltese ACHD patients to those of patients with similar conditions from other European countries, and uses data collected as part of the large scale multi-centre international project APPROACH-IS [10, 14].

Maltese patients had significantly poorer dental care and less regular sports participation when compared to their European counterparts. Poorer dental care was characterised by less regular dental reviews and lower rates of daily tooth brushing and dental flossing among Maltese patients with simple and moderately complex CHD. Dental care is particularly relevant in ACHD patients, especially those with mechanical and transcatheter valves, cyanotic disease or past history of infective endocarditis (IE), who are known to be at even higher risk of developing IE. Oral commensals are among the commonest microorganisms responsible for infective endocarditis (IE), particularly in individuals with poor oral hygiene [15]. Hospital activity analysis by the Directorate for Health Information and Research (Pieta', Malta) recorded 22 IE cases in 2014 and 28 cases in 2015, which represent 0.03 and $0.04 \%$ of all recorded patient hospital discharges nationwide, respectively.

Maltese ACHD patients claimed similar levels of dayto-day exercise, in the form of walking or cycling to work, when compared to their European counterparts, but participation in regular sports activities was significantly less among Maltese patients with simple and moderately complex lesions. Frequent and sustained physical activity has several benefits including vascular health, prevention of obesity and improvement in muscle function [16] as well as improvement in psychological, cognitive and social function [17]. On the other hand, a sedentary lifestyle is associated with a number of cardiac-related conditions including hypertension, diabetes, atherosclerotic disease and obesity [18].

Comparison of dental care and regular sports participation between all Maltese participants and participants from each European country was subsequently performed in an attempt to understand whether the trends noted among Maltese patients could be seen among other Europeans. Regular sports participation by Maltese participants was the lowest and the differences with other European countries were all strongly statistically significant suggesting that this tendency is quite unique among Maltese patients. Similarly, at least daily tooth brushing among Maltese patients was significantly lower than that seen in all other European countries. While Malta was comparable to France with regard to 
dental flossing and dental review practice, all other European countries had significantly better dental flossing practice and their patients were attending dental visits on a significantly more regular basis.

Hospital reviews represent an ideal setting for healthcare professionals to reinforce the importance of a healthy lifestyle with their patients. It could thus be argued that the poorer health-risk behaviour observed among Maltese patients with simple CHD could, at least in part, be explained by their less frequent clinical follow-up. However, this factor would not explain the tendency for poorer lifestyle noted in Maltese patients with moderately complex disease as their follow-up was comparable to that of other European patients with similar lesions. A study by Neinstein et al. suggests that risk perception could vary with age, with young adults tending to perceive risk less than adolescents and consequently tending to engage in more risky behaviour [19]. Based on this, it is possible that the younger age of Maltese participants in all three complexity groups could be one factor responsible for the differences in lifestyle habits observed. However, although Maltese patients were younger, their mean ages were $29.66 \pm 12.01$ years for simple CHD, $33.52 \pm 13.36$ years for moderate CHD and $29.59 \pm 5.23$ years for greatly complex CHD, meaning that most patients did not fit the 'young adult' category referred to in the study by Neinstein et al. [19]. It could also be hypothesised that 'sicker' patients might have better risk perception and thus have better appreciation of the importance of a healthy lifestyle to avoid certain complications. This aspect could partly explain the observed differences between Maltese participants in the simple and moderate complexity subgroups and their European counterparts, considering that, overall, European patients with moderate disease had undergone more cardiac procedures, more had needed ICD/PPM implantation and more Europeans with simple and moderate disease had documented cardiac arrhythmias. However, this same argument could be counterintuitive in that arrhythmias and ICD implantations are among the main clinical reasons why healthcare professionals might recommend patients to avoid sports activities [20]. Interestingly, Maltese patients with disease of great complexity were the most comparable to their European counterparts for all habits, including dental care and physical activity. Although there is no clear explanation for this observation, it could be postulated that this finding represents a much better knowledge of the underlying CHD and thus better perceived risk stemming from more frequent investigations and hospital visits.

It is the authors' opinion that the observed trends in dental care and sports participation among Maltese ACHD patients are most likely a representation of the general Maltese attitude towards both habits. This is supported by data from the national study referred to earlier [9].
Indeed, in the quoted study, $54.8 \%$ of the general Maltese population (non-ACHD) cohort admitted to a lack of dental reviews in the preceding 12 months, and, although, as a group, the ACHD cohort showed a significantly better dental review practice, there were still a high proportion of patients (39.1\%) that had not had a recent dental visit. Data on tooth brushing and flossing were not collected on that occasion as they were not available for the general population cohort. For the national study, physical activity levels were calculated using an internationally recognised model, in line with that used for the European Health Interview Survey 2008 [21]. Once again, although there was a significantly higher proportion of ACHD patients that undertook only low levels of physical activity (69.4\%), the proportion of non-ACHD Maltese subjects admitting to low physical activity levels was still high at $37.1 \%$. Furthermore, a recent nationwide study among school-aged children and teenagers in Malta confirmed around $40 \%$ of school attenders to be overweight or obese, a trend which is, at least in part, related to limited sports participation among children from an early age [22].

A better understanding of how well our patients appreciate their heart disease, possibly by using formal measures to assess disease knowledge, could help us gain insight into the reasons behind the uptake of unhealthier lifestyles [23, 24]. Misconceptions regarding safety of exercise in the presence of CHD, which are often known to be a main driver for poor physical activity and sports participation [25, 26], should be directly addressed and clarified during clinic visits and safe levels of exercise formally prescribed in line with international guidelines [20, 27]. The current study was performed before the implementation of a formal transition process for Maltese CHD patients, and it is thus hoped that, as younger patients go through this process, they grow up being better educated about their condition and more willing to embrace a healthier lifestyle attitude. The availability of clinical psychologists and specialist nurses with experience in the management of adolescents and adults with CHD could also improve adherence to a healthier lifestyle.

\section{Limitations}

Maltese ACHD patients participating in APPROACH-IS were expected to answer the English language version of the questionnaire, following an agreement with the steering committee. Although the majority of Maltese people have adequate English language proficiency, this fact might have excluded a proportion of patients who were unable to understand the questionnaire. This had the potential of excluding patients with lower levels of education, which in itself is a factor known to be related to unhealthier lifestyles. Malta had the lowest response rate among the 
participating European centres with 119/378 (31\%) invited subjects returning an answered questionnaire. The mean response rate for all participating European centres was $59 \pm 18 \%$ (range $31-92 \%$ ). The most likely explanation for this is the fact that, in an attempt to recruit the largest number of participants out of the small Maltese ACHD population, all patients in the national database that met eligibility criteria were invited. The invited group included an important number of patients with mild CHD that were no longer under regular specialist follow-up and who were thus not familiar with the research group contacting them. In fact, 22/295 non-participants formally declined participation by claiming it as "not relevant to their condition". While the low response rate in Malta could have introduced selection bias, it could conversely have selected out patients that were more likely to adopt habits similar to the general Maltese population. Thus, the final cohort consisted largely of patients that had more regular contact with specialist care.

\section{Conclusions}

This study has helped to highlight lifestyle aspects that Maltese ACHD patients need to improve on, which might not have been as evident by solely comparing patients to non-CHD controls from the same country. It suggests that, at least in Malta, CHD patients might still have a tendency to "follow the crowd" such that a concerted effort is required from an early age by all healthcare professionals involved to advocate healthier lifestyles. Such work also highlights the benefits of multi-centre collaboration, particularly in specialties like ACHD, where patient numbers are often small. Finally, our findings should caution researchers against considering trends and behaviours among patients in one country as representative of patients on the larger scale.

Acknowledgements The authors would like to thank Dr. Sandra Distefano (Consultant in Public Health Medicine, Directorate for Health Information and Research) for providing national statistics on infective endocarditis. APPROACH-IS consortium: Luis Alday, Héctor Maisuls, Betina Vega (Córdoba, Argentina, Hospital de Niños); Samuel Menahem, Sarah Eaton, Ruth Larion, Qi Feng Wang (Melbourne, Australia, Monash Medical Center); Werner Budts, Kristien Van Deyk (Leuven, Belgium, University Hospitals of Leuven); Silke Apers, Eva Goossens, Jessica Rassart, Koen Luyckx, Philip Moons (Leuven, Belgium, University of Leuven); Gwen Rempel, Andrew Mackie, Ross Ballantyne, Kathryn Rankin, Colleen Norris, Dylan Taylor, Isabelle Vondermuhll, Jonathan Windram, Pamela Heggie, Gerri Lasiuk (Edmonton, Canada, University of Alberta); Paul Khairy, Anna Proietti, Annie Dore, Lise-Andrée Mercier, François-Pierre Mongeon, François Marcotte, Reda Ibrahim, Blandine Mondésert, Marie-Claude Côté (Montreal, Canada, Montreal Heart Institute); Adrienne Kovacs, Erwin Oechslin, Mimi Bandyopadhyay (Toronto, Canada, University Health Network); Alexandra Soufi, Sylvie Di Filippo, François Sassolas, André Bozio (Lyon, France, Hospital Louis Pradel); Shanthi Chidambarathanu, Farida Farzana,
Nitya Lakshmi (Chennai, India, Frontier Lifeline Hospital, Dr. K. M. Cherian Heart Foundation); Edward Callus, Emilia Quadri, Massimo Chessa, Giovanna Campioni, Alessandro Giamberti (Milan, Italy, IRCCS Policlinco San Donato Hospital); Junko Enomoto, Yoshiko Mizuno (Chiba, Japan, Chiba Cardiovascular Center); Maryanne Caruana, Victor Grech, Sheena Vella, Anabel Mifsud, Neville Borg, Daniel Chircop, Matthew Mercieca Balbi, Rachel Vella Critien, James Farrugia, Yanika Gatt, Darlene Muscat (Msida, Malta, Mater Dei Hospital); Katrine Eriksen, Mette-Elise Estensen (Oslo, Norway, Oslo University Hospital); Mikael Dellborg, Malin Berghammer (Gothenburg, Sweden, Sahlgrenska University Hospital); Eva Mattson, Anita Strandberg, Pia Karlström-Hallberg (Stockholm, Sweden, Karolinska University Hospital); Bengt Johansson, Anna-Karin Kronhamn (Umeå, Sweden, University Hospital of Umeå); Markus Schwerzmann, Corina Thomet, Margrit Huber (Bern, Switzerland, University Hospital Bern); Jou-Kou Wang, Chun-Wei Lu, Hsiao-Ling Yang, Yu Chuan Hua (Taipei, Taiwan, National Taiwan University Hospital); Barbara Mulder, Maayke Sluman (Amsterdam, the Netherlands, Amsterdam Medical Center); Marco Post (Nieuwegein, the Netherlands, St. Antonius Hospital); Els Pieper (Groningen, the Netherlands, University Medical Center Groningen); Kathinka Peels (Eindhoven, the Netherlands, Catharina Hospital); Marc Waskowsky (Zwolle, the Netherlands, Isala Clinic); Gruschen Veldtman, Michelle Faust, Colin Lozier, Christy Reed, Jamie Hilfer (Cincinnati, USA, Cincinnati Children's Hospital Medical Center); Curt Daniels, Jamie Jackson (Columbus, USA, Nationwide Children's Hospital); Shelby Kutty, Carolyn Chamberlain (Omaha, USA, Children's Hospital \& Medical Center); Stephen Cook, Morgan Hindes (Pittsburgh, USA, Children's Hospital of Pittsburgh of UPMC); Ari Cedars, Kamila White (Saint Louis, USA, Washington University and Barnes Jewish Heart \& Vascular Center, University of Missouri); Anitra Rompfh, Susan Fernandes, Kirstie MacMillen (Palo Alto, USA, Stanford University).

Funding The APPROACH-IS project was supported by the Research Fund-KU Leuven (Leuven, Belgium) through grant OT/11/033; by the Swedish Heart-Lung Foundation (Sweden) through Grant No. 20130607; and by the University of Gothenburg Centre for Person-centred Care (Gothenburg, Sweden). In Malta, the work was supported financially by A.M. Mangion Ltd. (Luqa, Malta), Technoline Ltd. (Gzira, Malta) and Cherubino Ltd. (Gzira, Malta) which together covered the costs of printing and posting of the questionnaires.

\section{Compliance with Ethical Standards}

Conflict of interest The authors declare that they have no conflict of interest.

Ethical Approval All procedures performed in studies involving human participants were in accordance with the ethical standards of the institutional and/or national research committees of the participating centres and with the 1964 Helsinki declaration and its later amendments or comparable ethical standards.

Informed Consent Informed consent was obtained from all individual participants included in the study.

\section{References}

1. Moons P, Bovijn L, Budts W, Belmans A, Gewillig M (2010) Temporal trends in survival to adulthood among patients born 
with congenital heart disease from 1970 to 1992 in Belgium. Circulation 122(22):2264-2272

2. Pilapil M, DeLaet D (2015) Health risk behaviors in adolescents and young adults with special health care needs. Curr Opin Pediatr 27(1):132-137

3. Roche SL, Silversides CK (2013) Hypertension, obesity, and coronary artery disease in the survivors of congenital heart disease. Can J Cardiol 29(7):841-848

4. Zomer AC, Vaartjes I, Uiterwaal CS, van der Velde ET, Sieswerda GJ, Wajon EM et al (2012) Social burden and lifestyle in adults with congenital heart disease. Am J Cardiol 109(11):1657-1663

5. Moons P, Van Deyk K, Dedroog D, Troost E, Budts W (2006) Prevalence of cardiovascular risk factors in adults with congenital heart disease. Eur J Cardiovasc Prev Rehabil 13(4):612-616

6. Sandberg C, Pomeroy J, Thilen U, Gradmark A, Wadell K, Johansson B (2016) Habitual physical activity in adults with congenital heart disease compared with age- and sex-matched controls. Can J Cardiol 32(4):547-553

7. Janssens A, Goossens E, Luyckx K, Budts W, Gewillig M, Moons P (2016) Exploring the relationship between diseaserelated knowledge and health risk behaviours in young people with congenital heart disease. Eur J Cardiovasc Nurs 15(4):231-240

8. Overgaard D, Schrader AM, Lisby KH, King C, Christensen RF, Jensen HF et al (2014) Substance use, dental hygiene, and physical activity in adult patients with single ventricle physiology. Congenit Heart Dis 9(1):75-82

9. Caruana M, Grech V (2016) Lifestyle habits among adult congenital heart disease patients in Malta. Congenit Heart Dis 11(4):332-340

10. Apers S, Kovacs AH, Luyckx K, Alday L, Berghammer M, Budts W et al (2015) Assessment of patterns of patient-reported outcomes in adults with congenital heart disease-International Study (APPROACH-IS): rationale, design, and methods. Int $\mathbf{J}$ Cardiol 179:334-342

11. Goossens E, Luyckx K, Mommen N, Gewillig M, Budts W, Zupancic N et al (2013) Health risk behaviors in adolescents and emerging adults with congenital heart disease: psychometric properties of the Health Behavior Scale-Congenital Heart Disease. Eur J Cardiovasc Nurs 12(6):544-557

12. Warnes CA, Liberthson R, Danielson GK et al (2001) Task force 1: the changing profile of congenital heart disease in adult life (32nd Bethesda Conference). J Am Coll Cardiol 37(5):1170-1175

13. Ladouceur M, Iserin L, Cohen S, Legendre A, Boudjemline Y, Bonnet D (2013) Key issues of daily life in adults with congenital heart disease. Arch Cardiovasc Dis 106(6-7):404-412

14. Apers S, Kovacs AH, Luyckx K, Thomet C, Budts W, Enomoto J et al (2016) Quality of life of adults with congenital heart disease in 15 countries: evaluating country-specific characteristics. J Am Coll Cardiol 67(19):2237-2245
15. Habib G, Lancellotti P, Antunes MJ, Bongiorni MG, Casalta JP, Del Zotti F, et al (2015) ESC Guidelines for the management of infective endocarditis: The Task Force for the Management of Infective Endocarditis of the European Society of Cardiology (ESC). Endorsed by: European Association for Cardio-Thoracic Surgery (EACTS), the European Association of Nuclear Medicine (EANM). Eur Heart J 36(44):3075-3128

16. Sothern MS, Loftin M, Suskind RM, Udall JN, Blecker U (1999) The health benefits of physical activity in children and adolescents: implications for chronic disease prevention. Eur J Pediatr 158(4):271-274

17. King AC, Taylor CB, Haskell WL, DeBusk RF (1989) Influence of regular aerobic exercise on psychological health: a randomized, controlled trial of healthy middle-aged adults. Health Psychol 8(3):305-324

18. Ekelund U, Anderssen S, Andersen LB, Riddoch CJ, Sardinha LB, Luan J et al (2009) Prevalence and correlates of the metabolic syndrome in a population-based sample of European youth. Am J Clin Nutr 89(1):90-96

19. Neinstein LS, Irwin CE Jr (2013) Young adults remain worse off than adolescents. J Adolesc Health 53(5):559-561

20. Longmuir PE, Brothers JA, de Ferranti SD, Hayman LL, Van Hare GF, Matherne GP et al (2013) Promotion of physical activity for children and adults with congenital heart disease: a scientific statement from the American Heart Association. Circulation 127(21):2147-2159

21. Oja P (2003) Development of a common instrument for physical activity. In: Nosikov A, Gudex C (eds) EUROHIS-developing common instruments for health surveys. IOS Press, Amsterdam, pp 87-88

22. Grech V, Aquilina S, Camilleri E, Camilleri K, Busuttil ML, Sant'Angelo VF, Calleja N (2016) The Malta Childhood National Body Mass Index Study-A Population Study. J Pediatr Gastroenterol Nutr doi:10.1097/MPG.0000000000001430

23. Moons P, De Volder E, Budts W, De Geest S, Elen J, Waeytens K et al (2001) What do adult patients with congenital heart disease know about their disease, treatment, and prevention of complications? A call for structured patient education. Heart 86(1):74-80

24. Van Deyk K, Pelgrims E, Troost E, Goossens E, Budts W, Gewillig M et al (2010) Adolescents' understanding of their congenital heart disease on transfer to adult-focused care. Am J Cardiol 106(12):1803-1807

25. Swan L, Hillis WS (2000) Exercise prescription in adults with congenital heart disease: a long way to go. Heart 83(6):685-687

26. Reybrouck T, Mertens L (2005) Physical performance and physical activity in grown-up congenital heart disease. Eur J Cardiovasc Prev Rehabil 12(5):498-502

27. Budts W, Borjesson M, Chessa M, van Buuren F, Trigo Trindade P, Corrado D et al (2013) Physical activity in adolescents and adults with congenital heart defects: individualized exercise prescription. Eur Heart J 34(47):3669-3674 